ENCYCLOPÉDIE Encyclopédie berbère

BERBERE

$36 \mid 2013$

36| Oryx - Ozoutae

\title{
Ouargla [Wargrən $<$ Warglən] : Langue
}

Salem Chaker

\section{OpenEdition}

Journals

Édition électronique

URL : https://journals.openedition.org/encyclopedieberbere/2848

DOI : $10.4000 /$ encyclopedieberbere. 2848

ISSN : 2262-7197

\section{Éditeur}

Peeters Publishers

\section{Édition imprimée}

Date de publication : 2 juin 2013

Pagination : 5911-5920

ISBN : 978-2-7584-0194-0

ISSN : 1015-7344

\section{Référence électronique}

Salem Chaker, « Ouargla [Wargran < Warglən] : Langue », Encyclopédie berbère [En ligne], 36 | 2013,

document 036, mis en ligne le 12 mars 2021, consulté le 17 février 2022. URL : http://

journals.openedition.org/encyclopedieberbere/2848; DOI : https://doi.org/10.4000/

encyclopedieberbere. 2848

Ce document a été généré automatiquement le 17 février 2022.

(c) Tous droits réservés 


\title{
Ouargla [Wargrən $<$ Warglən] : Langue
}

\author{
Salem Chaker
}

1 Le dialecte berbère de Ouargla (təggargrent) regroupe en fait le parler des habitants anciens de la ville (ceux du ksar pour l'essentiel), mais aussi celui de N'goussa* (təggengusit) et de l'oued Righ* (tariyit/tarighit), parlé dans la région de Touggourt* (ksars de Ghomra, Meggarine, Temacine, Tamelhat et Blidet-Amor).

2 Il convient d'insister sur cette identification « habitants anciens »- on n'ose le terme « autochtones »!- car la population de Ouargla, capitale algérienne des hydrocarbures, a connu un profond renouvellement et une croissance exponentielle depuis la fin des années 1950 (actuellement autour de 135.000 habitants; cf. notice 035). Les berbérophones y sont devenus très largement minoritaires. Le recensement algérien de 1966, le seul qui ait comporté la rubrique "langue maternelle» depuis l'indépendance du pays, n'est pas utilisable pour Ouargla dans la mesure où les résultats accessibles sont globalisés par wilaya (département), en l'occurrence toute la wilaya des Oasis (i.e. à l'époque, la moitié du Sahara algérien!). Le répertoire «Ethnologue: Languages of the World», (SIL International) estime en 1995 à 5.000 les berbérophones de Ouargla ${ }^{1}$. J. Delheure, dans l'introduction à son corpus de textes (1988), estime la population berbérophone de l'ensemble Ouargla-Ngoussa à 10.000 personnes. Selon ses informateurs, en 1947, seul un cinquième de la population locale parlait le berbère, les autres étant arabophones (Delheure 1988, p. 20/21). Actuellement, les locuteurs du ouargli stricto sensu devraient représenter moins de $10 \%$ de la population de la ville (10 à 15.000 personnes sur 135.000).

3 La dénomination locale təggargrənt, "langue de Ouargla", provient du syntagme əw_wargrən, "fils/enfant de Ouargla", avec assimilation régulière $/ w+w />[g g]$ (əggargrən = homme de Ouargla), les noms de langues étant toujours au féminin en berbère. Le nom même de la ville, Wargrən/Warglən, est probablement construit sur une racine pan-berbère RGR < RGL, "fermer, obstruer, barrer..."; Delheure (1987 s.v. "Ouargla" et 1988a, p. 24, note 1) propose d'y voir un syntagme déterminant relatif *wa + (y)rgl-n, "celui (qui) est fermé, barricadé”, en référence au caractère défensif du site : 
«... ce ksar était bien défendu [...]. Il était, au moins jusqu'en 1930, encore entouré de remparts et surtout d'un large fossé plein d'eau stagnante... » (1987, p. 272). Ce type de dénominations construites autour d'un support de détermination (ici le défini wa, "celui") est effectivement bien attesté dans l'onomastique berbère (cf. Chaker, notice 017 "Onomastique libyco-berbère" et Galand 1974). Cette étymologie ne peut cependant être considérée comme sûre: les sources arabes médiévales donnent déjà une forme totalement figée dans laquelle le $y / i$ initial d'un éventuel participe irglen n'est jamais présent ; il en est de même pour la forme touarègue : Warglən. Par ailleurs, les sources arabes notent toujours un / $\bar{a} /$ long sur la première syllabe et très souvent aussi sur la dernière : wārğlā(n) (mais El-Bekri donne Wārğlan), ce qui constitue une petite difficulté et ouvre la voie à d'autres hypothèses, notamment à celle d'un groupe nominal privatif war + (i)gla/ən, «Sans +... (Nom pluriel) », modèle bien attesté en onomastique berbère (cf. 017).

4 Ce dialecte est perçu par ses locuteurs comme formant un ensemble avec celui du Mzab* (ou tumzabt) avec lequel l'intercompréhension est immédiate et dont, de fait, il est extrêmement proche. L'unité linguistique (et historique) de l'ensemble OuarglaMzab-Oued Righ, dénommé twargrit (Delheure 1987, p. 273 et 1988, p. 20-24) est explicitement perçue et affirmée par les locuteurs ouarglis :

Da ini Aylan, d awal iggən, biha da d aməzday-nsən i d-ffyən si-s = «Ici ou au Mzab, c'est la même chose, car c'est ici leur pays d'origine. » (Delheure 1988, p. 16/17)

At Wargrən fəhhmən d awəhdi tawsint, «Les Ouarglis comprennent très bien le tawsint [= parler de Témacine] » (Delheure 1987, p. 355).

5 La bibliographie sur le berbère de Ouargla est extrêmement concentrée ; les travaux récents de Jean Delheure: sa Grammaire de la Teggargrent... (1987b) - qui reste malheureusement difficile d'accès et mériterait une vraie édition et quelques développements -, son Dictionnaire Ouargli-Français (1987c), son imposant corpus de textes ethnographiques Vivre et mourir à Ouargla (1988a), et son recueil de Contes et légendes berbères de Ouargla (1989c), auxquels on rajoutera la série de textes littéraires qu'il a publiés (1987a, 1988b, 1989a, 1989c, 1990a, 1990b, 1992a, 1992b) constituent l'essentiel des connaissances solides sur la langue (et la littérature) ouarglie. On soulignera tout particulièrement l'apport essentiel de son Vivre et mourir à Ouargla (1988a) pour la connaissance de la société traditionnelle ouarglie. On mentionnera aussi travaux du R.P. Jardon (grammaire et corpus de textes : 1952, 1955, 1970, 1971), eux aussi difficiles d'accès. Avant ces travaux, qui émanent tous du Fichier de Documentation Berbère des "Pères Blancs" ${ }^{2}$, on peut se référer aux grands anciens, René Basset (1893), très vieilli maintenant, et surtout Samuel Biarnay (1908).

On signalera que la présence française dès 1852 (cf. notice 035) fit de la ville le commandement militaire français pour l'ensemble du Sahara algérien; Ouargla fut de ce fait, à maintes reprises, un poste d'observation et d'étude des populations berbères sahariennes et de leur langue : la plupart des berbérisants pionniers du XIX siècle y ont séjourné, notamment pour entrer en contact avec des Touaregs, caravaniers ou prisonniers de guerre, qui servirent de premiers informateurs (Hanoteau : 1860; Cid Kaoui : $1883 \% .$.$) .$

7 Comme le mozabite, le ouargli présente quasiment toutes les caractéristiques des parlers dits zénètes*. A la lumière de ces travaux, les principaux traits linguistiques du ouargli sont les suivants (tous les exemples proviennent de Delheure 1987 ou 1988a) : 


\section{Phonétique - Phonologie}

8 - Le système vocalique est de type "classique", avec trois voyelles phonologiques (/i, a, $\mathrm{u} /$ ), sans distinction de durée, et un schwa ([ə]) purement phonétique, servant d'appui syllabique. Le ouargli ne présente donc aucune spécificité par rapport à la phonologie des grands dialectes berbères Nord. Avec un système vocalique très réduit, les réalisations ont naturellement tendance a être fortement influencées par l'environnement phonétique, notamment avec ouverture et postériorisation des voyelles $(/ \mathrm{u} />[\mathrm{o}] ; / \mathrm{i} />[\mathrm{e}] ; / \mathrm{a} />[\alpha]>[\mathrm{o}])$ en contexte pharyngalisé ou vélaire, classiques mais particulièrement marquées en ouargli selon Delheure :

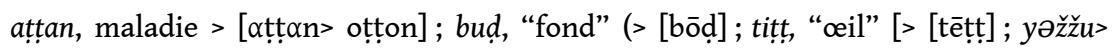

[yəžžō], « il a laissé »;

et aussi, rarement, sans conditionnement apparent : ass-u> [asso], « aujourd'hui ».

10 Cette tendance à la postériorisation semble avoir un pendant au niveau des consonnes liquides (cf. infra).

11 - La tendance, largement attestée dans tous les parlers "zénètes", à traiter les palato-

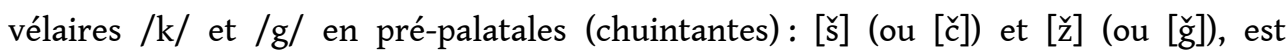
présente, mais elle est cependant bien moins marquée et généralisée qu'en mozabite :

nəธ̌̌̆, "moi" < nəkk; šəm "toi (fém.)" < kem

ašša, "demain" < azkka

aždid "oiseau" < agḍid,

amžər "faucille" < amgər.

12 Mais on a argaz "homme", tikli, "marche", alors que le mozabite présente arğaz, tičli...

13 - En revanche, absence totale de spirantisation* et d'affriction des dentales, contrairement à la plupart des parlers de l'Algérie du Nord, comme le chaoui (partiellement), le kabyle ou les parlers dits “zénètes" de l'Algérie centrale (Chenoua*, Ouarsenis*...) et occidentale.

14 - La labiovélarisation est rare (mais moins qu'au Mzab); elle est attestée pour les labiales $\left[\mathrm{b}^{\mathrm{w}}\right],\left[\mathrm{f}^{\mathrm{w}}\right]$, la nasale $\left[\mathrm{m}^{\mathrm{w}}\right]$, les palato-vélaires sonore $\left[\mathrm{g}^{\mathrm{w}}\right]$ et sourde $\left[\mathrm{k}^{\mathrm{w}}\right]$, l'uvulaire $\left[\mathrm{q}^{\mathrm{w}}\right]: t f^{w} i$, soleil ; $\mathrm{mm}^{w} a$, (mon) frère $(</ \mathrm{u}-\mathrm{ma} /)$; asəgg ${ }^{w} a s$, "année"; aməqq ${ }^{w}$ ran, "grand». Dans de nombreux cas, elle résulte de phénomènes d'assimilation aux frontières de morphèmes (préposition $+w^{-}$de l'Etat d'Annexion* du nominal subséquent), phénomène bien connu dans de nombreux dialectes berbère Nord :

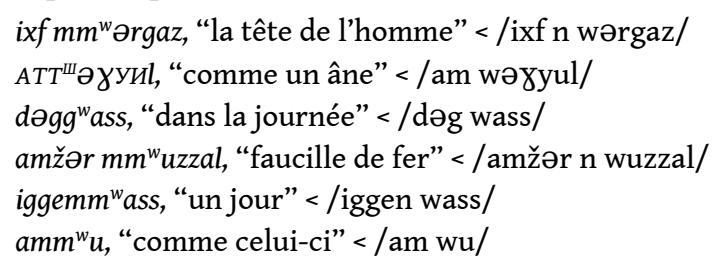

15 - Plus spécifique à Ouargla est la tendance marquée à la confusion des constrictives dentales (en fait pré-alvéolaires) et prépalatales : /s/-/̌s/ et $/ z /-/ \check{z} /$. Delheure (p. 6/7) fait état d'un flottement permanent chez la plupart des locuteurs, à l'exception des familles ibadites des Ayt Waggin, sans doute en raison de liens plus étroits avec le Mzab :

təžžu uššu tẓiwa alterne librement avec təzzu ussu tẓiwa, "elle a laissé du couscous dans le plat";

išəmžan, "nègres, esclaves noirs", alterne avec išəmzan et isəmzan (forme

"berbère" : isəmgan). 
16

note une tendance assez nette à la vélarisation/pharyngalisation des liquides (/l/ $/ \mathrm{r} /, / \mathrm{n} /$ et $/ \mathrm{m} /$ ). Certains cas paraissent spontanés (sans conditionnement détectable) : ar, "ouvrir" (cf. touareg-Ahaggar ar) ; tamurt, "pays" (cf. kabyle tamurt). Mais la plupart sont issus de phénomènes d'assimilation anciens, bien attestés ailleurs (notamment en touareg) de séquences "liquides+phonèmes vélarisants" (surtout $/ \mathrm{\gamma} /$ ); un cas

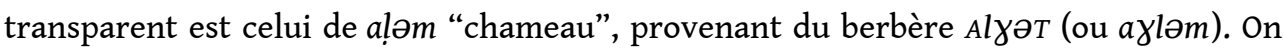
aboutit ainsi à de véritables - mais rares - paires minimales fondées sur les oppositions /l/ /1/, /n/ /n/, /m/ /m/ :

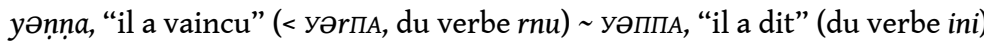

17 le phénomène soit généralisé et stabilisé comme dans la majorité des parlers rifains ; en ouargli, cette évolution paraît relever d'une tendance à l'harmonie consonantique au profit de /r/ lorsque le radical comporte deux liquides :

Wargrən < Warglən ; rwər alterne avec rwəl ("fuir, s'enfuir") ; tragda alterne avec

tragla ("clavicule, anneau d'attache").

18 - En finale absolue le / $/$ / (constrictive vélaire sonore) peut se vocaliser en [a] (sans doute $[\bar{a}])$, dans le lexique comme pour les morphèmes grammaticaux :

ura, "or" < urəy.

19 L'indice de personne de $1^{\text {ère }}$ personne $d u$ singulier $-\gamma$ subit normalement cette vocalisation, en finale absolue après consonne; il réapparaît sous sa forme consonantique si le verbe est suivi d'un affixe grammatical quelconque :

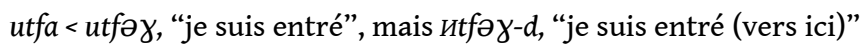

$x s a<x s \partial \gamma$, "je veux", mais $x s \partial \gamma-t$, "je le veux"

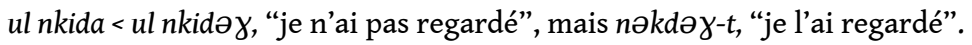

20

- Comme en mozabite, la tendance à la chute de la voyelle initiale du nom, dans certains contextes phonologiques (noms de forme (v)CvC...), caractéristique des parlers zénètes, est bien représentée :

dar < adar, "pied"; fus < afus, "main" ; yanim < a a anim, "roseau"; zaw < azaw,

"cheveu" ; yazid < ayazid, coq...

\section{Morphosyntaxe}

\section{Système du Nom}

21

- Le morphème de pluriel pré-nominal id (cf. notice 1117, «Ida », EB XXIV, 2001) est bien attesté à Ouargla devant certains nominaux sans flexion : nanna, "mère" > id-nanna "mères" ; bab, "maître" > id-bab, maîtres.

- Autre trait caractéristique des parlers zénètes, le déictique de proximité (suffixe nominal) a une forme $-u$ (et $-y u$ en position post-vocalique), au lieu du "classique" $-a$ (kabyle, chleuh...) : argaz-u, cet homme, taddart-u, cette maison ; middən-u “ces gens”. D’où les déictiques autonomes $w u(w-u)$, «celui-ci » et $t u(t-u)$, « celle-ci », en face de wa et ta, du kabyle, touareg, etc.

24 - La série des pronoms personnels affixes du nom (" possessifs ») présente, au singulier, les formes primitives courtes (sans $n$ ) :

Encyclopédie berbère, 36 | 2013 


\begin{tabular}{|l|l|l|l|}
\hline$-(i)_{w}$ & mon/ma/mes & $-n-n a .(</ n-n ə \gamma /)$ & notre/nos \\
\hline$-ə) k$ & ton/ta/tes & $-n-k u m$ & votre/votre \\
\hline$-(ə)_{T}$ & ton/ta/tes & $-n-k u m t$ & votre/vos (fém.) \\
\hline$-(ə)_{s}$ & son/sa/ses & $-n$-sən & leur \\
\hline & & $-n$-sənt & leur (fém.) \\
\hline
\end{tabular}

- Les pronoms affixes du verbe de la série directe montrent à la $3^{\mathrm{e}}$ pers. du sing. deux particularités remarquables:

Le très classique - $t$ "le" se vocalise parfois totalement en $-i$, et la forme de féminin ("la"), qui a la forme complète -tət, en -it; d'où des énoncés :

sərs-i (<sərs-t), "pose-le !" et sərs-it (<sərs-tət), "pose-la !"

ce qui n'est pas sans rappeler un phénomène parallèle de coalescence/vocalisation en

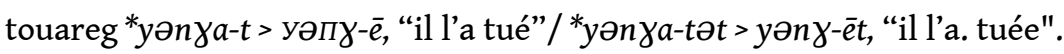

\section{Le système verbal}

- Survivance, apparemment plus marquée qu'en mozabite, d'un thème d'aoriste intensif négatif (ou inaccompli négatif), trait lui aussi largement répandu dans le groupe des parlers zénètes (Kossman 1989). Ce thème conditionné par la négation $u l / u$, est présent pour plusieurs classes importantes de verbes; il est toujours marqué par une voyelle fermée /i/ alternant avec la voyelle ouverte /a/ :

xəs (A) / qqas (AI) / qqis (AIN), "vouloir"

af (A) / taf (AI) / tif (AIN), "trouver"

bədd (A) / tbədda (AI) / tbəddi (AIN), "être debout"

z̧un (A) / tżuna (AI) / tzuni (AIN), "partager"

$\operatorname{arr}$ (A) / ttərra (AI) / ttərri (AIN), "rendre"

29 - Vocalisation en $/ u$ / de la $3^{\mathrm{e}}$ personne du singulier ("il/elle") et de la $1^{\text {ère }}$ du pluriel ("nous") au prétérit (accompli) de la plupart des verbes mono-et bi-consonantiques à alternance finale $(\varnothing / \mathrm{i}-\mathrm{u})$, en face du /a/ kabyle, chleuh ou touareg :

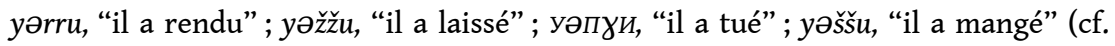

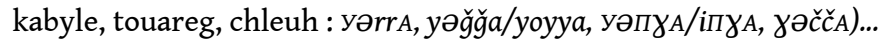

30 - Le thème de prétérit (négatif) en /i/ est bien maintenu pour toutes les classes morphologiques de verbes qui le connaissent habituellement: CCC, CC, C, aCC, aC..., etc. :

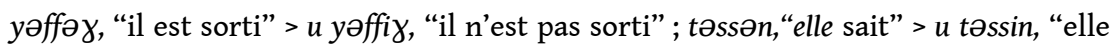
ne sait pas"; уәпуи, "il a tué" > u уәпүi, "il n'a pas tué" ; yənkəd, "il a regardé"> u yənkid, "il n'a pas regardé".

31 - L'aoriste a les emplois bien connus ailleurs :

32 En tant que forme non marquée dans le récit, dans une séquence verbale située par le premier verbe (succession d'actions « enchaînées ", Bentolila 1981, §4.14-4.44):

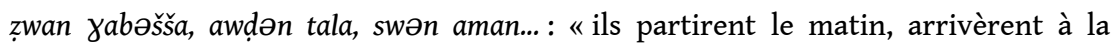
source, burent de l'eau... » 
Précédé du préverbe ad, il prend, comme en kabyle, en touareg..., les valeurs temporelles et modales, de futur, potentiel, injonctif, optatif... :

ašša ad ffyən, "demain, ils sortiront"

ad alint, "(qu') elles montent !" (certainement avec un intonation spécifique, non signalée par Delheure).

xsa ad ffyən, "je veux (qu') ils sortent"...

34 - La négation verbale a la forme $u l, u$ (ou $w$ devant voyelle), sans second élément de renforcement, selon une distribution, apparemment simple, morphologiquement conditionnée :

ul devant thème verbal nu (i.e. sans morphème préfixé) : ul nkida, "je n’ai pas regardé"; ul ššin, "ils n'ont pas mangé",

$u$ devant thème verbal commençant par un affixe quelconque (Indice de personne ou morphème satellite) : "u yə犭ris, "il n'a pas égorgé"; u yenkid, "il n'a pas regardé"; u tenkid "elle n’a pas regardé" ; u t-nkida, "je ne l'ai pas regardé".

- Le ouargli connaît les deux particules d'orientation*, $d$ (orientation vers le locuteur) et $n$ (éloignement/orientation vers l'auditeur) : awi-d, "apporte (ici)!". Mais $n$ est beaucoup plus rare et n'apparaît guère qu'avec des formes défectives, interjectives comme iyya-n, "viens !" (Delheure 1987, p. 103-108).

- Le ouargli, à l'instar de la plupart des dialectes Nord et de son voisin mozabite, utilise de nombreux verbes auxiliaires, à valeur temporelle, aspectuelles et/ou modales (cf. Chaker 1997); parmi les plus courants et les plus intéressants par leur distribution géographique très large, on citera :

ili, "être", marquant la concomitance : llan ttarin, "ils sont en train d'écrire"; ssi ddusin lliy ttəff za, "je sortais quand ils vinrent"

$k k ə r$, "se lever", à valeur inchoative : yəkkər yəttusu, "il se mit à tousser"

әxs "vouloir", à valeur d'imminence : yəxs ad yəmmət, "il est sur le point de mourir"

ttuð, préverbe totalement grammaticalisé à valeur temporelle de passé révolu...

\section{Syntaxe}

- Présence et usage fréquent, comme dans tous les parlers berbères " méditerranéens ", de l'auxiliaire de prédication nominale $d$ (+ Nom à l'état libre) :

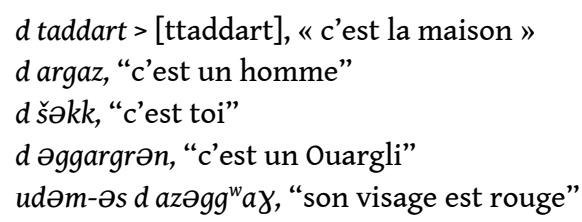

D'autres types de phrase nominale sont possibles, utilisant des auxiliaires de prédication non spécifiques (cf. Chaker 1983, chap. 22 à 26 et 1984, chap. 8), notamment la prépositions $n$, "de" :

awal nn-iw "la parole est à moi" (" c'est mon tour de parler »),

39 - La négation de phrase nominale utilise un morphème ugi, manifestement issu du

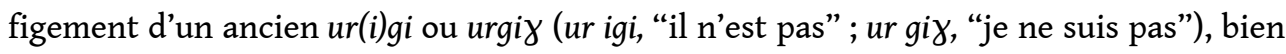
connu en touareg et qui renvoie à un usage ancien du verbe-copule əg, "faire/être fait/ être" :

ugi sən, "ce n'est pas deux / ils ne sont pas deux" 
- Le parler de Ouargla est caractérisé par la perte de fonction très marquée de l'Etat d'annexion* (EA) du nom ; Delheure évoque « ...un abandon progressif de la marque u-/ wa de l'Etat d'annexion des noms masculins...» (p. 54). La marque d'EA semble ainsi avoir disparu dans le contexte syntaxique où, dans les autres dialectes, il conserve sa pertinence maximale en assurant la distinction entre le complément d'objet (marqué par l'Etat Libre) et le complément explicatif (= sujet lexical post-posé, marqué par EA) ; on relève ainsi des énoncés surprenants par rapport au kabyle, au chleuh ou au touareg :

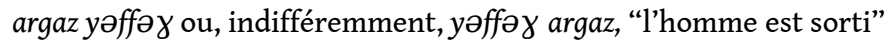

yəzwa argaz, "l'homme est parti" (au lieu de urgaz/wərgaz).

41 Le principal contexte d'apparition de l'Etat d'annexion du nom reste donc le syntagme nominal prépositionnel, ce qui tend à faire de l'EA un pur phénomène morphologique, conditionné par la présence d'une préposition (cf. El Moujahid 1982).

42 - Comme dans la généralité du berbère à l'exception du kabyle, la série des interrogatifs, est fondée sur la base chamito-sémitique ma : matta, "quoi?", mani, "où?", mammu, "qui ?"...

43 - Si dans l'usage courant, la numération* a été intégralement empruntée à l'arabe, on note que les familles ibadites des Ayt Waggin connaissent encore l'intégralité de l'ancienne numération berbère (Delheure 1987, p.57-58), situation particulière qui renvoie là encore certainement à des liens plus étroits avec le Mzab ibadite.

\section{Texte}

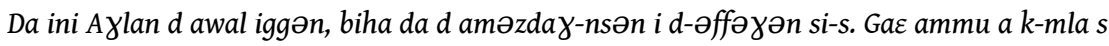
mənnawt iwalən azur n At-Mzab-u. Middən-u, a Sidi, t-tarwiwin $n$ Ibadiyən i rəwrən $s$ Tihart, sagga tən-d-zəckən imalkiyən s təgəldit-nsən. Usin $n$ da ammas $n$ Șșhəhrt, ḥban

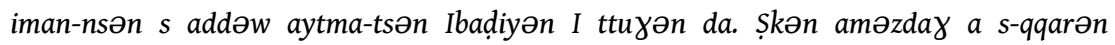
Isədratən s addəw ugerbub a s-nəqqar nəšnin "Tżiwa n Iعəzzabən"

"Ici ou au Mzab, c'est la même chose, car c'est ici leur pays d'origine. A ce propos, il faut que je te raconte en bref l'origine de ces Mozabites. Ces gens-là, Cher Monsieur, sont les descendants des Ibadites qui s'enfuirent de Tahert lorsqu'ils furent chassés de leur empire par les Malékites. Ils vinrent ici se réfugier en plein désert auprès de leurs coreligionnaires qui s'y trouvaient déjà. Ils édifièrent une ville appelée Sedrata, au voisinage de la colline-témoin que nous désignons sous le nom de "Plat à cousus des Ibadites" ». (Delheure 1988, p. 16/17, collecté en 1947).

\section{BIBLIOGRAPHIE}

BASSET R., 1893 - Etude sur la zénatia du Mzab, de Ouargla et de l'Oued Rir', Paris, Leroux (Publ. Ecole des Lettres d'Alger - Bulletin de Correspondance Africaine).

BENTOLILA F., 1981 - Grammaire fonctionnelle d'un parler berbère, Paris, SELAF, 447 p..

BIARNAY S., 1908 - Etude sur le dialecte berbère de Ouargla, Paris, Leroux, 501 p. 
BISSON J., 2003 - Mythes et réalités d'un désert convoité, Paris, L'Harmattan, 480 p.

BLANCHET P., 1900 - «L'oasis et le pays de Ouargla », Annales de Géographie, 1900, t. 9, nº 44.

p. 141-158.

CHAKER S., 1983 - Un parler berbère d'Algérie (Kabylie) : Syntaxe, Aix/Marseille, UNIVERSITÉ de Provence/Jeanne Lafitte, 549 p. +111 p.

CHAKER S., 1984 - Textes en linguistique berbère (Introduction au domaine berbère), Paris, Editions du CNRS, 1984, $291 \mathrm{p}$

CHAKER S., 1997 - «Quelques faits de grammaticalisation dans le système verbal berbère », Mémoires de la Société de Linguistique de Paris, n.s., V, ("Grammaticalisation et reconstruction »), p. 103-121.

DEJEUX J., 1978 - Djoh'a hier et aujourd'hui, Paris-Montreal-Sherbrooke, Naaman,.

[Corpus inédit de contes de Ouargla.]

DELHEURE J., 1987a - « Id akkaz ini id ahazi. Devinettes et énigmes de Ouargla Sud algérien) », Etudes et Documents Berbères 3, p. 76-83.

DELHEURE J., 1987b - Grammaire de la Teggargrent berbère parlé à Ouargla, Paris, Pères Blancs, 143 p.

DELHEURE J., 1987c - Dictionnaire Ouargli-Français. Agerraw $n$ iwalen teggargrenttarumit, Paris, SELAF, 493 p. (Maghreb-Sahara: 5).

DELHEURE J., 1988a - Vivre et mourir à Ouargla. Tameddurt $t$-tmettant wargren, Paris, SELAF, $436 \mathrm{p}$. (Maghreb-Sahara : 7).

DELHEURE J., 1988b - « Baghdidis et l'ogre. Saynète dans le parler de Ouargla », Etudes et Documents Berbères 4, p. 103-115.

DELHEURE J., 1989a - « Izlan d-id aghanni. Poésies et chants de Ouargla », Etudes et Documents Berbères 5, p. 85-104.

DELHEURE J., 1989b - « Systèmes verbaux ouargli-ouargli », Journée d'Etudes de Linguistique Berbère, Paris, Publications Langues'O, p. 53-64.

DELHEURE J., 1989c - Contes et légendes berbères de Ouargla. Tinfusin, Paris, La Boîte à Documents, $403 \mathrm{p}$.

DELHEURE J., 1990a - «Proverbes et dictons de Ouargla », Etudes et Documents Berbères 7, p. 111-135. DELHEURE J., 1990b - «Id irar Wargren - Jeux à Ouargla », Awal, Cahiers d'études Berbères, 6, p. 291-298.

DELHEURE J., 1992a - «Id irar Wargren - Jeux à Ouargla (suite) », Awal, Cahiers d'études Berbères Alger, Bouchène), 8, p. 145-161.

DELHEURE J., 1992b - «Calendrier, Calendrier agraire de Ouargla Mzab) », Encyclopédie berbère, 11, Aix-en-Provence, Edisud, p. 1717-1719.

DELHEURE J., REESINK P., 1973 - « Le morphème $n$ en ouargli, préposition et/ou connecteur, Le Fichier Périodique [FDB], 119, p. 48-73.

EL MOUJAHID H., 1982 - « Un aspect morphologique du nom en tamazight : l'état d'annexion », Langues et Litteratures (Rabat), 2. 
FDB : Fichier de Documentations Berbère, Fort-National (Algérie) : nombreux textes avec traductions (voir « Tables chronologiques et Index », par J. Lanfry, Alger, Le Fichier Périodique [FDB], 124, 1974/ IV). Ceux de M. Jardon sont regroupés dans JARDON 1970 et 1971.

GALAND L., 1974 - « Défini, Indéfini, Non-défini : les supports de détermination en touareg », BSLP, LXIX/1, p. 205-224.

JARDON M., 1952 - Le verbe ouargli. Formes simples... Wargren (Ouargla), Fort-National (Algérie), FDB, $35 \mathrm{p}$.

JARDON M., 1955 - « Le cheval à six pattes. Tanfust m-bu-setta n-yidaren. Ouargla », Texte et trad., Fort-National (Algérie), FDB 46, 20 p.

JARDON M., 1970 - Ouargla I, Textes berbères, Transcrits, traduits et annotés par J. Delheure, FortNational (Algérie), FDB, $192 \mathrm{p}$.

JARDON M., 1971 - Ouargla II, Le mariage à Ouargla, Transcrits, traduits et annotés par J. Delheure, Fort-National (Algérie), FDB, 538 p.

KoSSMANN M. 1989 - «L'inaccompli négatif en berbère », Etudes et Documents Berbères 6, p. 19-29.

LE THIELLEUX J., 1983 - Ouargla, cité saharienne. Des origines au début du $20^{\mathrm{e}}$ siècle, Paris, Geuthner, $298 \mathrm{p}$.

OULD-BRAHAM O., 1988 - « Sur une chronique arabo-berbère des Ibadites médiévaux », Etudes et Documents Berbères 4, p. 5-28.

ROMEY A., 1975 - « Toponymie et culture orale d'une oasis de la ceinture préprésaharienne : Ngoussa », Libyca, XXIII, p. 243-256.

ROMEY A., 1992a - « Toponymie et culture en milieu saharien berbérophone », Lettre d'Information de l'Association Française pour l'Etude du Monde Arabe et Musulman (Paris), 7, p. 112-113.

ROMEY A., 1992b - Histoire, mémoire et sociétés. L'exemple de N'Goussa, oasis berbérophone du Sahara Ouargla, Paris/Alger, L'Harmattan/Awal, 176 p. [version publiée d'une thèse de 1974, Paris EPHE, $\mathrm{VI}^{\mathrm{e}}$ section].

ROUVILlOIS-BRIGol M., MESSON C., VALLET J., 1973 - Oasis du Sahara Algérie : de l'Oued Righ, Ouargla, Tamentit, Paris, Institut Géographique National, VII p. + 118 p.

ROUVILLOIS-BRIGOL M., 1975 - Le pays de Ouargla (Sahara alghérien). Variations et organisation d'un espace rural en milieu désertique, Paris, Sorbonne (Département de Géographie), 390 p.

SIGWARTH G., 1944 - «Appellations en langue ouargli du chameau aux différents stades de sa croissance », Bulletin d'Etudes Arabes (Alger), p. 139.

\section{NOTES}

1. Mais on restera en général circonspect devant les évaluations de cette organisation chrétienne américaine, qui ne fournit pas ses sources et n'a strictement aucun moyen propre de procéder à des enquêtes sur place. Beaucoup des chiffres qu'elle avance pour les variétés de berbère paraissent approximatifs, voire manifestement erronés.

2. L'ordre chrétien des Missionnaires d'Afrique ("Pères blancs") a été présent sur place à partir de 1873. 
INDEX

Mots-clés : Linguistique, Sahara, Zénète 